Mihailov Claudia, Tica Irina,

\title{
Autoimmune Thyroiditis In A Population With Rheumatoid Arthritis
}

Ovidius University, Faculty of Medicine Constanţa, IIIrd Medical Department

\begin{abstract}
Introduction: Autoimmune thyroiditis (AIT) associates a multitude of osteoarticular and muscular complaints but, in the same time, the association between AIT and rheumatoid arthritis (RA) remains a matter of controversy.

Material, patients and method: We performed a retrospective cross-sectional study over 69 patients with RA admitted successively in the the IInd Medical Clinic of Railway Hospital between 01 Jan 2015 and 01 Aug.2015, subdivided into two groups: with and without AIT. A number of parameters were studied in each group: age, environment, parity and number of gestations, smoker status, education level, medium duration of disease, presence of rheumatoid factor (RF), of anti-cyclic citrullinated protein antibodies (ACPA), erythrocyte sedimentation rate (ESR) and C-reactive protein (CRP) levels, treatment for RA, VAS score, DAS 28 score.

Conclusions: The percentage of patients with RA and AIT is $17.391 \%$, higher than in general population, and so we consider that screening for AIT in patients with RA should be done. Patients with RA
\end{abstract}

Tica Irina

University "Ovidius" of Constanta, Faculty of Medicine

Universitatii Street, No. 1, Campus B

Constanta, Romania

email: irinatica@gmail.com and AIT have a younger age of RA onset, belong to the urban environment and have a higher level of education than the ones with RA but without AIT. No correlation between the occurrence of AIT and the presence of ACPA was found.

Keywords: Autoimmune thyroiditis, rheumatoid arthritis, age, education, environment

\section{Introduction}

Autoimmune thyroiditis (AIT) associates a multitude of osteoarticular and muscular complaints. In the past, most of these manifestations were attributed to the underlying thyroid dysfunction, more frequently to hypothyroidism which is induced by AIT [4], but further research revealed that a multitude of antibodies is associated with AIT [1], many of them non-organ specific, which can be involved in the occurrence of these articular manifestations.

The association between rheumatoid arthritis (RA) and thyroid specific autoimmunity, with or without hormonal dysfunction, is present in $6 \%$ to $33.8 \%$ patients, most of them being females [2].

Even if the presence of characteristic organ specific antibodies in serum can precede even with a few years symptoms' onset and making a diagnosis [2] this can not be considered a reliable early sign 
of AIT because the antibodies are also found in low concentrations in healthy individuals [2].

Age, duration and activity of RA, the presence of rheumatoid factor and antinuclear antibodies have not been found to predispose to coexistence of these diseases [2]. The only factor established till now by medical literature to predispose to association between RA and AIT is represented by the the genetic background.

Today guidelines of endocrinology (both American and European) recommend that routine population screening for thyroid diseases must be performed only in risk groups of thyroid diseases development, (i.e women with exposure to radiotherapy or radiations, with family history of thyroid diseases, with previous thyroid dysfunction, with symptoms suggestive of thyroid dysfunction, with abnormalities in physical examination of the thyroid gland, type 1 diabetes and a history of other autoimmune diseases) [2].

Under these circumstances, the AIT diagnosis might be difficult to be established due to the fact that in the initial phase most of the patients are asymptomatic. This induces the search of the factors that could identify individuals particularly prone to the development of these diseases.

The purpose of our study was to determine the AIT prevalence in patients with RA in a specific population in our District and whether these patients should be screened for thyroid diseases.

\section{Material, patients and method}

Thestudy was typecross-sectional retrospective. Consecutive patients with RA admitted to the IInd Medical Clinic of Railway Hospital between 01 Jan 2015 and 01 Aug.2015 were investigated.

A group of 69 patients with RA were diagnosed on the basis of 2010 ACR/EULAR RA Classification Criteria (American College of Rheumatology. This group was divided into two other subgroups: patients with AIT (12 patients) and patients without AIT (57 patients)

In all the patients a follow up chart was done containing:

1. Clinical examination: i.e. medical history taking (including history regarding thyroid diseases, RA), physical examination with internal examination and musculoskeletal system assessment (28 joints assessment according to EULAR criteria), and evaluation of goiter and its grade according to World Health Organization.

Activity of RA was evaluated using VAS and the Disease Activity Score (DAS 28) with 4 parameters.

2. Laboratory tests used to evaluate RA activity: erythrocyte sedimentation rate, $\mathrm{C}$-reactive protein (CRP); and anti-cyclic citrullinated protein antibodies (ACPA) and tests of occurrence of autoimmune thyroid disease: antithyreoglobulin (anti-TGB) antibodies and antithyroid peroxidase (anti-TPO) antibodies. They were determined in the same outclinic laboratory (the same for all patients). The reference values for anti-TGB antibodies were $<$ $115 \mathrm{UI} / \mathrm{ml} 2$ and for anti-TPO antibodies $<34 \mathrm{UI} / \mathrm{ml} 2$

3. Thyroid ultrasound was not routinely performed because of its limited availability. It was performed in the Department, in 5 RA patients.

4. AID diagnosis was made based on the presence of antithyroid antibodies (presence of antiTPO antibodies was a prerequisite for the diagnosis) in patients with concomitant thyroid dysfunction and/ or goiter. ATD was suspected based on the values of anti-TPO antibodies close to the limits of the reference range and positive anti-TGB antibodies.

\section{Results and discussion}

The features of our study groups are registered in Table I and II. 
Table I Features of patients with RA

\begin{tabular}{|l|l|l|l|}
\hline & RA & RA without AIT & RA with AIT \\
\hline & $69(100 \%)$ & $57(82.608 \%)$ & $12(17.391 \%$ \\
\hline Age (min, max) (years) & 28,83 & 36,83 & $28, \ldots 63$ \\
Average (years) & 58.681 & 60.315 & 50.916 \\
Standard deviation & 12.210 & 12.246 & 8.86 \\
\hline $\begin{array}{l}\text { Sex } \\
\text {-Male }\end{array}$ & 5 & 5 & \\
-Female & 64 & 52 & 0 \\
\hline Life environment & & & 12 \\
-urban & $51(73.92 \%)$ & $41(71.93 \%)$ & $10(83.34 \%)$ \\
-rural & $18(26.08 \%)$ & $16(28.070 \%)$ & $2(16.66 \%)$ \\
\hline $\begin{array}{l}\text { Smoker status } \\
\text {-Negative }\end{array}$ & & & \\
-Positive & & & $1(8.33 \%)$ \\
\hline Medium duration of disease (years) & 5.47 & 5.36 & 5.29 \\
Standard deviation & 2.198 & 1.04 & 4.081 \\
\hline Level of education & & & \\
-Primary school and gymnasium & $21(30.43 \%)$ & $17(29.82 \%)$ & $2(16.66 \%)$ \\
-High school, apprentice school & $31(44.92 \%)$ & $26(45.61 \%)$ & $7(58.33 \%)$ \\
-Faculty, college & $17(24.63 \%)$ & $14(24.56 \%)$ & $3(25 \%)$ \\
\hline RF & & & \\
-Positive & $44(63.76 \%)$ & $36(63.15 \%)$ & $6(50 \%)$ \\
-Negative & $25(36.23 \%)$ & $21(36.84 \%)$ & $6(50 \%)$ \\
\hline MTX & $44(63.76 \%)$ & $38(66.666 \%)$ & $6(50 \%)$ \\
\hline SSZ & $5(7.24 \%)$ & $3(5.26 \%)$ & $2(16.6 \%)$ \\
\hline Rituximab & $12(17.39 \%)$ & $11(19.29 \%)$ & $1(8.33 \%)$ \\
\hline Etanercept & $15(21.73 \%)$ & $12(21.05 \%)$ & $3(25 \%)$ \\
\hline Adalimumab & $10(14.49 \%)$ & $7(12.28 \%)$ & $3(25 \%)$ \\
\hline Leflunomide & $18(26.08 \%)$ & $18(31.57 \%)$ & 0 \\
\hline AINS & $4(5.79 \%)$ & $2(3.50 \%)$ & $2(16.66 \%)$ \\
\hline Plaquenil & 0 & 0 & $2(16.66 \%)$ \\
\hline Concomitent thyroid disorders & $16(23.18 \%)$ & $4(7.01 \%)$ & $12(100 \%)$ \\
\hline & & &
\end{tabular}

$\mathrm{RF}=$ rheumatoid factor, DAS 28= Disease Activity

Score, $\mathrm{VAS}=$ Visual Analog Scale, ESR=erythrocyte sedimentation rate, $\mathrm{CRP}=\mathrm{C}$ reactive protein

AIT associates with a big variety of rheumatic manifestations attributed in particular to hypothyroidism [4]. However, there is evidence that some rheumatic manifestations are more frequent in hypothyroid patient with AIT than in those without this disease or they may occur even in euthyroid patients, supporting the idea of involvement of autoimmunity rather than a direct action of thyroid hormones [4].

Rheumatic manifestations could be sometimes attributable to the autoimmune rheumatic diseases frequently associated with AIT, such as systemic lupus erythematosus, rheumatoid arthritis, Sjögren's syndrome, or scleroderma. Among the most important or frequent rheumatic manifestations there are polyarthralgia, myalgia, a mild non-erosive variety of arthritis and sicca syndrome without a true Sjögren's syndrome. Possible hypothesis for the pathogenesis of these manifestations, yet incompletely known, include the role of autoantibodies characteristic of autoimmune thyroiditis, a possible overlap between AIT and some autoimmune rheumatic diseases, and a systemic inflammatory reaction associated with thyroiditis [4].

Table II. Patients with RA and with AIT

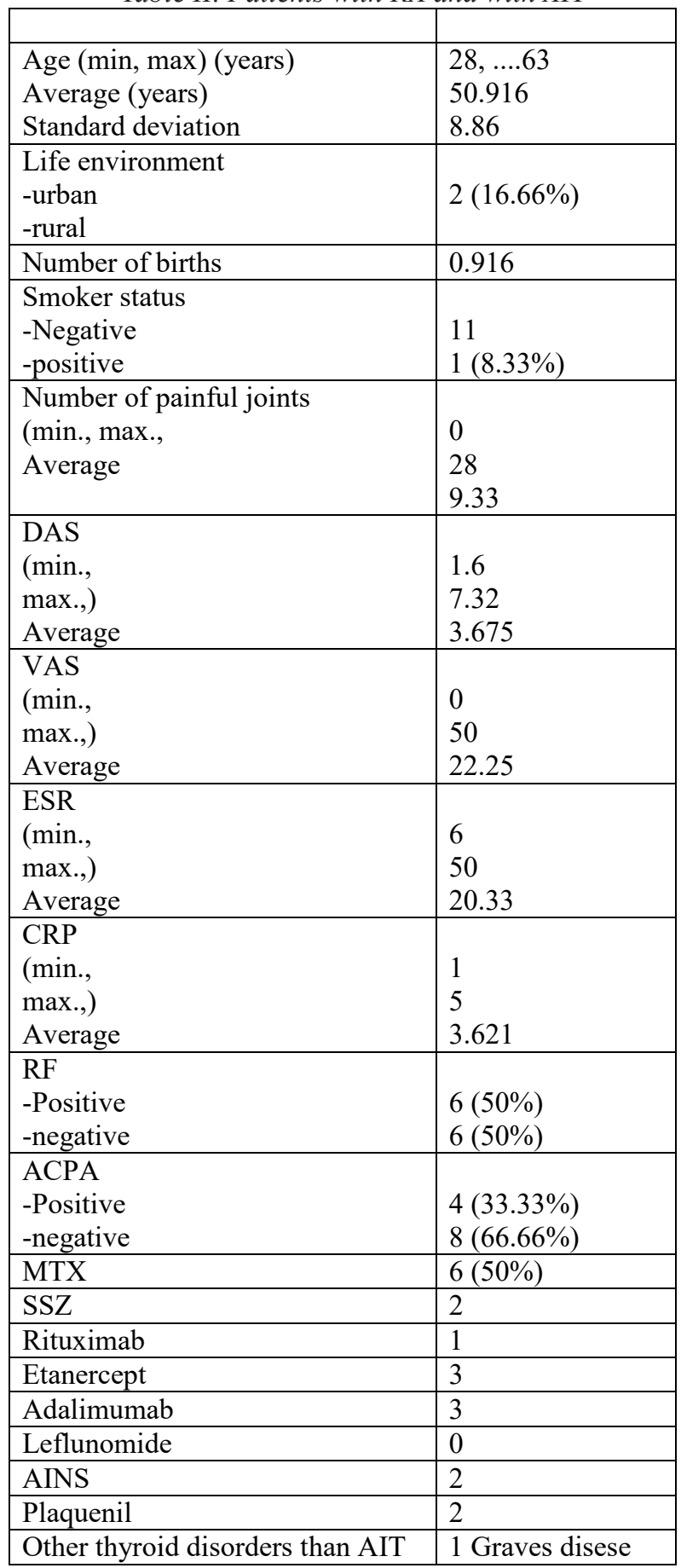

$\mathrm{RF}=$ rheumatoid factor, $\mathrm{ACPA}=$ anti-cyclic citrullinated protein antibodies, DAS 28= Disease Activity Score, $\mathrm{VAS}=$ Visual Analog Scale, $\mathrm{ESR}=$ erythrocyte sedimentation rate, $\mathrm{CRP}=\mathrm{C}$ reactive protein 
There are anyway studies that did not find a correlation between the two entities. One controlled population based study did not identify an association between thyroiditis and RA, but can be considered inconsistent as it is a retrospective chart review with an unclear case definition. Two further studies, one retrospective at necropsy and the other a small clinical study, did not identify a strong association [11].

In spite of these results, the relation between RA and AIT was noted in many situations, studies in several centers noting that thyroid disease is common in family members of patients with RA, with a genetic association apparently independent of HLA-DR4 [11]. Although the exact pathogenic mechanisms of the coexistence of autoimmune disorders are still not completely defined, genetics, immune defects, hormones and environmental factors may play key roles in autoimmunity, especially CTLA-4 and PTPN22 polymorphisms. [3]. Environmental factors influencing AIT development are iodine intake, drugs (iodine, L-2 treatment, lithium, amiodarone) infections (hepatitis $\mathrm{C}$ infection, parvovirus, rubella, herpes simplex virus, Epstein Barr virus, and human T-lymphotropic virus type 1, Toxoplasma gondii) and different chemicals (polyaromatic hydrocarbons or polyhalogenated biphenyls, polychlorinated biphenyls, living in areas of petrochemical complexes) [8].

There is little information available on the effect of stress on AIT, but some studies consider that there is evidence for an increase in postpartum thyroiditis, following the cellular immune suppressive effect of pregnancy [9], while other consider that stress is not involved in the pathogenesisi of AIT [10].

In view of this, we began a retrospective crosssectional survey of our patients with RA for the presence of thyroid dysfunction.

The percentage of patients with RA and AIT in our group of patients with RA is $17.391 \%$ that is higher than in general population and it fits within the limits revealed by medical literature. Literature data reveal that the prevalence of AITD in the general population varies between countries, $0.1 \%$ and $5 \%$ of all adults in Western countries [13], a prevalence of 5 to $15 \%$ in women and $1-5 \%$ in men [5]. The prevalence of thyroid autoantibodies has also been described. Hollowell et al. [6] described a prevalence of $13 \%$ for anti-TPO antibodies and $11.5 \%$ for antiTGB antibodies among the general population. This prevalence rises in spontaneously hypothyroid patients [7]. The range of AIT in RA has its lower limit at $0.5 \%$ and it goes up to $27 \%$ [12].

Our study revealed that the patients with RA and AIT have a younger medium age (50.19 years) than the ones with RA without AIT (60.315 years), probably because the association of the two diseases either augmented the severity of musculoskeletal symptoms or determined a younger onset of the diseases and so, an earlier diagnosis.

Another result of our study is the total feminine distribution of RA with AIT. This is similar to literature data, the female patients being at higher risk of developing these diseases than men.

The majority (83.34\%) of our patients belong to the urban environment, confirming the involvement of stress, pollution in the plurifactorial etiology of these disorders.

Also the patients with RA associating AIT had a higher level of education, almost $84 \%$ attending high school or college while only $70.17 \%$ of the patients of the group with only RA attended the same level of education.

The presence of rheumatoid factor was less present in our group with RA and AIT than in the one with RA without AIT, similar to the study of Przygodzka $M$ and al. in Poland

Regarding the presence of ACPA medical data is controversial also. Charles et al. [14] did not found a relationship between the presence of thyroid antibodies and the occurrence of ACPA although they did with PTPN22R620W allele. Kang et al. [15] found that in Korean patients with AR subclinical or clinical hypothyroidism was associated to the occurrence of positive titers of ACPA but with no conclusion related to AIT presence. In our study we did not find a correlation between AITD and proxy variables for RA severity such as biologic agent use or the presence of ACPA.

The percentage in which the patients with RA and AIT received DMARDs, respectively biologic therapy did not differ from the one in patients with RA but without AIT.

In the subgroup with RA and AIT the medium value of ESR was $20.33 \mathrm{div} / \mathrm{h}$ while the medium value 
of CRP was $3.621 \mathrm{mg} / \mathrm{dl}$. DAS 28 score revealed a moderate disease activity in patients with RA and AIT while the VAS assessment had a medium value of 22. It has to be specified that the patients were under treatment for at least 5 years, the medium disease duration in this subgroup being 5.29 years, the vast majority of patients being treated from the moment they were diagnosed.

\section{Conclusions}

1. The percentage of patients with RA and AIT in our group of patients is higher than in general population, and so we consider that screening for AIT in patients with RA should be done.

2. Patients with RA and AIT have a younger age of RA onset and in their majority belong to the urban environment, confirming the environmental involvement in both AIT and RA occurrence;

3. Patients with RA associating AIT have a higher level of education, and this condition sustains the idea of stress involvement in occurrence of both disorders.

4. We did not find any correlation between the presence of AIT and the presence of ACPA.

\section{References}

1. Benhatchi K., Lazúrová I., Kozáková D. \& Rovenský J. (2010). Prevalence of rheumatic manifestations and non-organ specific autoimmunity in patients with autoimmune thyreopathy. Vnitr Lek et. Feb,56(2):106-10.

2. Przygodzka M. \& Filipowicz-Sosnowska A. (2009). Prevalence of thyroid diseases and antithyroid antibodies in women with rheumatoid arthritis. Polskie Archiwum Medycyny
Wewnętrznej; 119 (1-2)

3. Lazúrová I., Jochmanová I., Benhatchi K. \& Sotak S. (2014). Autoimmune thyroid disease and rheumatoid arthritis: relationship and the role of genetics, Immunol Res. Dec; 60(2-3):193200. DOI: $10.1007 / \mathrm{s} 12026-014-8598-9$.

4. Punzi L. \& Betterle C. (2004). Chronic autoimmune thyroiditis and rheumatic manifestations. Joint Bone Spine. Jul; 71(4):27583

5. Dayan C.M. \& Daniels G.H. (1996). Chronic autoimmune thyroiditis. The New England Journal of Medicine, 335(2), 99-107.

6. Hollowell J.G., Staehling N.W., Flanders W.D. \& al. (2002). Serum TSH, T4, and thyroid antibodies in the United States population (1988 to 1994): national health and nutrition examination survey (NHANES III). Journal of Clinical Endocrinology and Metabolism,87(2), 489-499,.

7. Carle A., Laurberg P., Knudsen N. \& al. (2006). Thyroid peroxidase and thyroglobulin autoantibodies in patients with newly diagnosed overt hypothyroidism. Autoimmunity. 39(6), 497-503,

8. Zaletel K. \& Gaberšček S. (2011). Hashimoto's Thyroiditis: From Genes to the Diseas. Curr Genomics. 12(8): 576-588.

9. Tsatsoulis A. (2006). The Role of Stress in the Clinical Expression of Thyroid Autoimmunity. Annals of the New York Academy of Sciences, vol. 1088, Neuroendocrine and Immune Crosstalk pages 382-395.

10. Effraimidis G., Tijssen J.G.P., Brosschot J.F. \& Wiersinga W. M. (2012). Involvement of stress in the pathogenesis of autoimmune thyroid disease: A prospective study. Psychoneuroendocrinology. 37(8), Pages 1191-1198

11. Jeffrey B.S., Martin C., Marie-Louise B. \& Carolyn N. (1993). Thyroid dysfunction in rheumatoid arthritis: a controlled prospective survey. Annals of the Rheumatic Diseases. 52, 454-456

12. Jorge C.R. (2012). Autoimmune Thyroid Disease in Rheumatoid Arthritis: A Global Perspective, Arthritis, 2012(2012), 15 pages, DOI:10.1155/2012/864907

13. Gherbon A. (2014) Epidemiological aspects 
of autoimmune chronic thyroiditis in a group of adults with thyroid diseases and diabetes mellitus and other changes in glycemic balance. European Scientific Journal.10(21).

14. Charles P.J., Plant D., Chowdhury M., Worthington J. \& Venables P. (2011). Antibodies to thyroglobulin and thyroid peroxidase in rheumatoid arthritis: environmental and genetic associations. Annals of the Rheumatic Diseases. 70(2),A88-A89,
15. Kang E. J., Choi S. T., Park Y. B. \& S. Lee K., (2010) Thyroid disease in Korean patients with rheumatoid arthritis. International Journal of Rheumatic Diseases. 13,81-82,. 